\title{
Sífilis la gran imitadora. Reporte de caso y revisión de la literatura
}

\author{
Syphilis: the great imitator. A case report and literature review \\ Síflis o grande imitador. Relato de caso e revisão da literatura
}

Yadira V. Boza Oreamuno ${ }^{1}$ iD 0000-0002-0367-8664
Sandra M. Boza Oreamuno² (iD 0000-0001-6623-4924

\section{Resumen}

La sífilis es una enfermedad de transmisión sexual causada por el Treponema pallidum. Sin tratamiento es crónica y altamente contagiosa. Sus variadas manifestaciones clínicas dificultan su diagnóstico. Presentamos el caso de una lesión sifilítica en cavidad oral con alta sugerencia clínica de carcinoma de células escamosas.

Caso clínico: hombre de 31 años que presenta gran úlcera en mucosa labial inferior derecha de bordes indurados, 2 meses de evolución, asociada a dos adenopatías en cuello. Se realiza una minuciosa anamnesis y exámenes de laboratorio, confirmando el diagnóstico de una infección treponémica. Se le brindó tratamiento y la lesión resolvió. Los títulos de seguimiento fueron no reactivos.

Conclusiones: Se demuestra la importancia de mantener sospecha de infección sifilítica cuando un paciente presenta linfoadenopatía y una lesión oral, fundamentar un diagnóstico correcto proporciona una pronta atención y recuperación del paciente, evitando complicaciones que afecten su calidad de vida y a sus parejas sexuales.

Palabras clave: Sífilis. Cavidad Oral. Enfermedades de transmisión sexual. Treponema pallidum.

Departamento de Ciencias Diagnósticas y Quirúrgicas, Facultad de Odontología, Universidad de Costa Rica, Costa Rica.

Departamento de Análisis Clínicos, Facultad de Microbiología, Universidad de Costa Rica, Costa Rica. sandra.boza@ucr.ac.cr

Fecha de recibido: 28/07/2020 - Fecha de aceptado: 13/01/2021 


\section{Abstract}

Syphilis is a sexually transmitted disease caused by Treponema pallidum. Without treatment, it is chronic and highly contagious. Its varied clinical manifestations make it challenging to diagnose. We present the case of a syphilis oral lesion with a strong clinical suggestion of squamous cell carcinoma.

Clinical case: a 31-year-old man with a large ulcer in the right lower lip mucosa with indurated edges, two months of evolution, associated with two enlarged neck lymph nodes (lymphadenopathy). A thorough history is taken, and laboratory tests are run, confirming the treponemal infection diagnosis. He was treated, and the injury resolved. Follow-up tests were nonreactive.

Conclusions: It is important to suspect a syphilitic infection when a patient has lymphadenopathy and an oral lesion. Making the correct diagnosis allows for the proper care and recovery, avoiding complications that affect the patient's quality of life and sexual partners.

Keywords: Syphilis. Oral cavity. Sexually transmitted diseases. Treponema pallidum.

\section{Introducción}

La sífilis es una enfermedad de transmisión sexual (ETS) causada por una bacteria llamada Treponema pallidum, cuyo único huésped natural es el ser humano ${ }^{(1,2)}$. Su principal sitio de inoculación son los órganos genitales aunque, debido a las nuevas prácticas sexuales, esta bacteria tiene tropismo por las áreas de la cavidad oral y región anal ${ }^{3-5}$. También se puede dar transmisión de la madre al feto durante el embarazo o durante el parto ${ }^{(6,7)}$. Existe un alto riesgo de infección al

\section{Resumo}

A sífilis é uma doença sexualmente transmissível causada por Treponema pallidum. Sem tratamento, é crônico e altamente contagioso. Suas variadas manifestaçóes clínicas dificultam seu diagnóstico. Apresentamos o caso de uma lesão sifilítica na cavidade oral com uma alta sugestão clínica de carcinoma de células escamosas.

Caso clínico: homem de 31 anos de idade, com úlcera grande na mucosa do lábio inferior direito, com bordas endurecidas, 2 meses de evoluçāo, associado a linfadenopatia do pescoço. Uma anamnese completa e exames laboratoriais são realizados, confirmando o diagnóstico de uma infecção treponêmica. $\mathrm{O}$ tratamento foi realizado e a lesáo resolvida. Os títulos de acompanhamento não foram reativos.

Conclusóes: A importância de manter uma suspeita de infecção sifilítica quando um paciente apresenta linfadenopatia e uma lesão oral é demonstrada, justificando um diagnóstico correto, proporcionam atenção e recuperação imediatas do paciente, evitando complicaçóes que afetam sua qualidade de vida e seus parceiros sexuais.

Palavras-chave: Sífilis. Cavidade oral. Doenças sexualmente transmissíveis. Treponema pallidum.

compartir jeringas por el uso de drogas ${ }^{(8)}$. Pocos casos de transmisión por transfusiones sanguíneas han sido reportados ${ }^{(1,8)}$.

Para la Organización Mundial de la Salud (OMS) la sífilis es considerada un problema de salud pública a nivel mundial, formando parte de las ocho ETS con mayor incidencia ${ }^{(7)}$.

Como la aparición de síntomas es muy variable y esta enfermedad cursa con periodos prolongados de latencia, la mayoría de las veces las personas ni siquiera se dan cuenta que tienen sífilis. El desconocimiento, conlleva a la propa- 
gación de la enfermedad, motivo por el cual es una infección tan importante de diagnosticar y tratar ${ }^{(9)}$.

La sífilis sin tratamiento es una enfermedad crónica ${ }^{(10,11)}$. El tratamiento de elección es la penicilina $\mathrm{G}$ benzatina, la dosis y la duración, dependerán del estadio de la enfermedad y de las manifestaciones clínicas del paciente ${ }^{(5,10)}$.

El objetivo de este trabajo es presentar el caso de una lesión sifilítica en cavidad oral con alta sugerencia clínica de carcinoma de células escamosas, y evidenciar la importancia de un diagnóstico preciso y rápido, ya que así, aumenta la eficacia de la estrategia de tratamiento no solo para el paciente sino para las parejas sexuales del mismo y evitar más contagios.

\section{Antecedentes}

Los síntomas varían en las diferentes etapas; la sífilis primaria se manifiesta generalmente por una única úlcera (chancro) en el lugar de la inoculación, se da generalmente entre 9 a 90 días luego del periodo de incubación y tarda aproximadamente 3 semanas para desaparecer espontáneamente, aunque se han reportado periodos hasta de 8 semanas ${ }^{(3,5,12)}$. El chancro es indoloro, altamente contagioso y puede pasar desapercibido ${ }^{(5,13)}$.

Si no se la trata, la enfermedad progresa a la etapa secundaria que puede causar una erupción cutánea que aparece en diferentes partes del cuerpo, entre 2 y 12 semanas luego de desaparecer el chancro (en algunos casos aún está presente) ${ }^{(1,14)}$. Es muy indicativo de sífilis secundaria el observar eritemas en las palmas de las manos y plantas de los pies ${ }^{(5,13)}$. Además, se pueden observar una variedad de lesiones en boca, las cuales, son más comunes en esta fase que en la primaria ${ }^{(3)}$.

Se le ha llamado "la gran imitadora" porque la sífilis tiene tantos síntomas posibles, muchos de los cuales se parecen a los de otras enfermedades ${ }^{(15)}$. Las lesiones en la mucosa oral son bastante variables y simulan patologías como liquen pla- no, úlcera eosinofílica, linfoma, sialometaplasia necrotizante y carcinoma oral de células escamosas (COCE) ${ }^{(16)}$.

La etapa terciaria se manifiesta como úlceras perforantes que reciben el nombre de goma sifilítico que pueden culminar en una neurosífilis o sífilis cardiovascular ${ }^{(11,14)}$.

La infección puede tener periodos de latencia (ausencia de síntomas y/o signos) entre las etapas secundaria y terciaria, en los que se diagnostica únicamente por pruebas serológicas ${ }^{(17)}$.

\section{Descripción del caso}

Hombre de 31 de años, nicaragüense, privado de libertad que es traído a la Facultad de Odontología de la Universidad de Costa Rica (UCR) con la queja principal de lesión en labio inferior de aproximadamente 2 meses de evolución. En el interrogatorio directo no reporta antecedentes ni contraindicaciones sistémicas. No toma medicamentos actualmente. Refiere ser fumador crónico de 30 cigarrillos al día desde los 16 años. Al preguntar por su actividad sexual refiere ser soltero, bisexual y reconoció prácticas de sexo oral con varones no protegidas.

Al realizar el examen clínico se observó bermellón inferior reseco y fisurado, a nivel de mucosa labial inferior derecha úlcera de 3 x 4 $\mathrm{cm}$, bordes muy indurados asociados a zonas erosivas y fondo cubierto por una seudomembrana amarillenta, dolorosa a la palpación y al roce con los dientes, refiere que inició más pequeña, pensó que se había mordido o lastimado, pero no sanó más bien ha crecido en 2 meses (Fig. 1A). En cuello en el nivel III derecho presentaba dos adenopatías de 2 $\mathrm{cm}$, tipo ovoide y dolorosas a la palpación, tiempo de evolución dos días. No se observaron alteraciones de contorno facial ni en piel (Fig. 1B). 
Fig. 1: A. Úlcera en mucosa labial inferior derecha, de $3 \times 4 \mathrm{~cm}$, bordes indurados asociados a zonas erosivas y fondo cubierto por una seudomembrana amarillenta. B. Dos adenopatías de $2 \mathrm{~cm}$, tipo ovoide en el nivel III de cuello lado derecho.
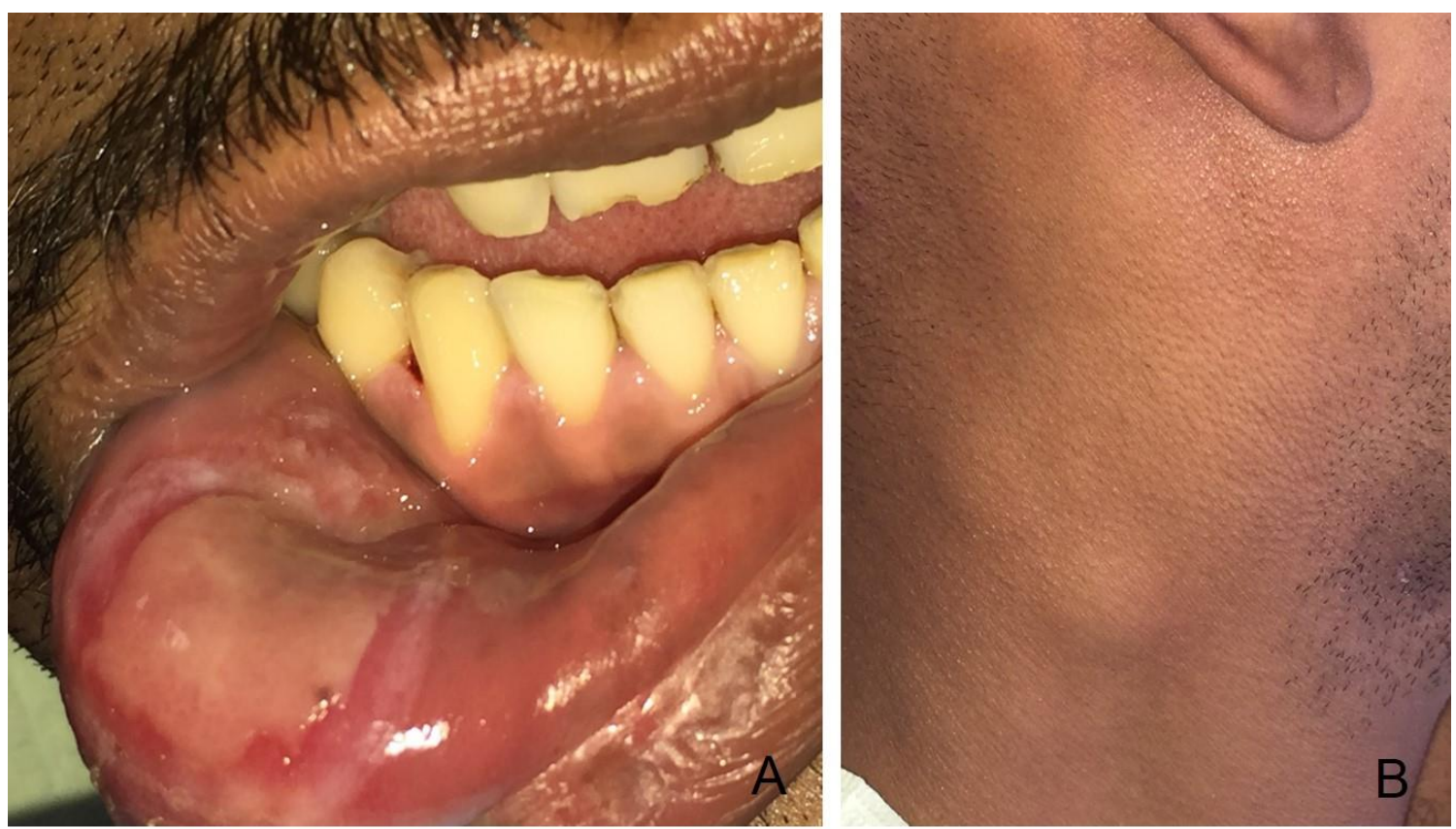

Tomando en cuenta los hallazgos encontrados se establece el diagnóstico clínico de COCE vs chancro sifilítico; se procedió a enviar ultrasonido (US) de cuello con biopsia por aspiración con aguja fina (BAAF) y exámenes de laboratorio: hemograma completo, VDRL (por sus siglas en inglés, Venereal Disease Research Laboratory) y virus de inmunodeficiencia humana (VIH).

Resultados del US cuello presencia de dos adenopatías en el nivel III miden $22 \mathrm{~mm}$ y $18 \mathrm{~mm}$, con ausencia de hilio y BAAF negativa por células malignas, compatible con adenopatía reactiva. Pruebas de sangre VIH negativo, VDRL positivo, se le realizó FTA-Abs (fluorescente treponemal antibody absortion) y el resultado fue positivo.

Con el diagnóstico definitivo de sífilis fue referido a la Caja Costarricense del Seguro Social y le enviaron tratamiento con penicilina $2.4 \mathrm{mi}-$ llones de unidades, intramuscular dosis cada 8 días por 3 semanas. También se le solicitó que todas sus parejas se realizaran el examen de sangre para descartar sífilis.
La lesión se curó dentro de una semana de tratamiento (Fig. 2). Se realizó seguimiento con examen de sangre VDRL negativo a los 3, 6, 12 y 24 meses, refirió funcionalidad bucal y confort, está libre de lesiones.

Fig. 2: Control a los 8 días de iniciado el tratamiento donde se observa ya sana la zona donde estaba la lesión

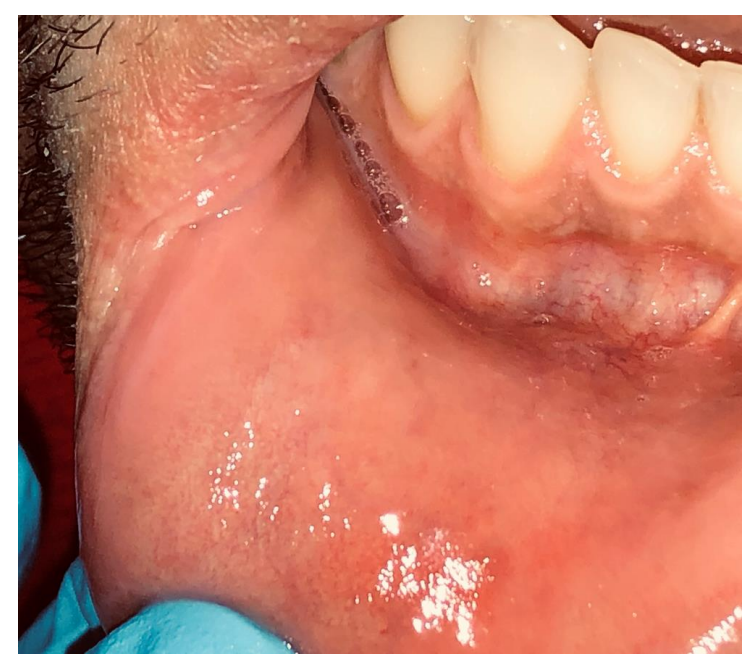


El paciente brindó por escrito su consentimiento libre e informado para la publicación del caso.

\section{Discusión}

Se ha observado un incremento en la tasa de casos de sífilis reportados en todo el mundo ${ }^{(9)}$, según la OMS con un estimado de 5.6 millones de casos nuevos cada año ${ }^{(18)}$. En nuestro país el grupo etario más afectado es el de 20-65 años y según género el masculino es el que presenta mayor número de casos ${ }^{(19)}$.

Se ha reportado que las poblaciones de riesgo son hombres que mantienen relaciones sexuales con otros hombres, las trabajadoras del sexo y sus clientes, personas que tienen numerosas parejas sexuales, transgénero o que ya tienen una ETS $(7,20,21)$. Por otro lado, existen poblaciones vulnerables, como lo son los adolescentes, niños y jóvenes que viven en las calles, presos y consumidores de drogas ${ }^{(7)}$. Lo antes descrito, concuerda con nuestro caso donde el paciente era un hombre, privado de libertad, teniendo sexo con hombres y mujeres.

El Treponema pallidum entra por la mucosa o piel erosionada y se empieza a dividir en el lugar de inoculación produciendo la sífilis primaria (5). Después de un periodo de incubación que de promedio dura 21 días (rango entre 9 y 90 días) ${ }^{(11)}$; aparece la lesión primaria, un chancro indurado e indoloro, de base limpia, bordes firmes y sobre elevados, siendo rico en treponemas. Generalmente se acompaña de linfadenopatía lo$\mathrm{cal}^{(1)}$ o regional ${ }^{(13)}$. En nuestro caso el chancro sifilítico se localizaba en mucosa labial inferior, por lo que la inoculación se debió a práctica de sexo oral; el paciente estaba asintomático, pero presentaba adenopatía no supurativa en cuello. Por la apariencia de nuestro caso debemos recordar su similitud con el COCE, el cual, ha sido considerado un cáncer más ligado a los varones, se ha reportado que más del $90 \%$ de los pacientes con cáncer bucal han sido fumadores (22). En estadios avanzados puede presentarse como un tumor grande con o sin ulceraciones, úlcera profunda con una superficie vegetante irregular, bordes elevados y un infiltrado duro de los tejidos bucales; propenso a la rápida diseminación hacia los nodos linfoides y a la metástasis ${ }^{(23)}$. Lo anterior concuerda con el caso en estudio, es decir, clínicamente alta sospecha de un COCE; por lo cual es de suma importancia la habilidad del entrevistador para hacer diagnóstico diferencial con sífilis.

Para un diagnóstico temprano de sífilis, se necesitan detalles de la historia sexual y del uso de drogas, información que las personas no suelen brindar de manera voluntaria y fácil ${ }^{(24)}$. Un buen examen físico, en el cual hay conocimiento de las manifestaciones clínicas, es de gran importancia al igual que las pruebas de laboratorio que se utilicen y su debida interpretación para completar el diagnóstico de sífilis ${ }^{(2)}$.

Entre las pruebas de escrutinio o no treponémicas, están RPR (Rapid Plasma Reagin) y VDRL. Estas pruebas son fáciles y rápidas de realizar. Su gran valor está en que pueden distinguir entre una infección activa y una anterior, pero su desventaja es su baja especificidad en ciertas etapas de la enfermedad, llevando a falsos positivos $(6,11,17)$. Por esta razón, se requiere de una prueba confirmatoria, entre las cuales están CMIA (chemiluminescence immunoassay), HTPA (Treponema pallidum hemaglutination) y APTP o FTA-Abs, debido a que estas pruebas detectan anticuerpos, permanecen positivas para toda la vida y no distinguen una infección activa de una tratada, por tal razón deben realizarse en conjunto con una prueba presuntiva $(6,17)$. En nuestro caso el VDRL y el FTA-ABS fueron las pruebas diagnósticas de sífilis que se utilizaron.

Se recomienda que, hombres que tienen sexo con otros hombres, se realicen la prueba anualmente y, si hay más factores de riesgo asociados, como numerosas parejas o sexo combinado con drogas ilícitas, realizar la prueba cada 3 meses $(13,24)$. 
Debido a que clínicamente puede confundirse con diferentes estados patológicos de la cavidad bucal tales como tuberculosis oral, ulceraciones aftosas, linfomas, sialometaplasia necrotizante, COCE, entre otros ${ }^{(16,25-27)}$, y sobre todo cuando la lesión es el único signo de la enfermedad puede ser necesario realizar una biopsia, en la cual, un infiltrado inflamatorio rico en células plasmáticas debe sugerir el diagnóstico, lo que induce al estudio inmunohistoquímico con anticuerpo anti-Treponema, donde es posible observar las espiroquetas para confirmar sífilis ${ }^{(28,}$ ${ }^{29)}$. Así mismo, es de utilidad el uso de BAAF de los nodos linfoides afectados, que en nuestro caso permitió descartar malignidad y confirmar una adenopatía reactiva, que sumada a las pruebas serológicas se obtuvo el diagnóstico definitivo de sífilis.

La Penicilina G parenteral es la droga de elección en todas las etapas de la enfermedad dado que la eficacia es máxima y no existe resistencia ${ }^{(10)}$. Es responsabilidad del profesional en salud, dar seguimiento al tratamiento con la intención de ver la respuesta del paciente al mismo; así como dar tratamiento a las parejas sexuales del paciente que ha tenido en los últimos 90 días $(20,30)$. Si las parejas sexuales son más allá de los 90 días, se recomienda realizarles las pruebas de laboratorio para confirmar si están infectadas o no; en caso afirmativo, dar tratamiento ${ }^{(10,20,30)}$. En nuestro caso ambas partes fueron abordadas. La práctica del sexo oral y el uso de drogas ilícitas ha ido en aumento, sobre todo entre los adolescentes, probablemente como una manera segura de evitar embarazos, sin preocuparse o tener la información adecuada sobre los riesgos y las con- secuencias de las lesiones orales altamente infecciosas ocasionadas por las ETS ${ }^{(4,11,24,31)}$.

La OMS tiene en curso (periodo 2016-2021) una estrategia mundial para disminuir radicalmente las ETS y las muertes relacionadas a estas (7). La meta para el 2030 es reducir en un $90 \%$ la incidencia de sífilis a nivel mundial (32). Para ello, se requiere que cada país defina las poblaciones específicas más afectadas, además de contar con apoyo político y recursos para mantener las medidas de una manera sostenible ${ }^{(7,33)}$.

Este reporte de caso resulta importante porque se diagnosticó de forma temprana la sífilis. Además, es un claro ejemplo que el trabajo en equipo con otros especialistas del área de la salud es fundamental.

\section{Conclusiones}

Es de suma importancia que los profesionales del área de la salud y en especial los odontólogos se familiaricen con esta patología. Se demuestra la importancia de mantener sospecha de infección sifilítica cuando un paciente presenta linfoadenopatía y una lesión oral.

El rápido diagnóstico y manejo correcto en nuestro caso permitió una pronta atención y recuperación del paciente, así como a sus parejas sexuales evitando que se infectaran más personas y se afectara su calidad de vida.

El asesoramiento puede mejorar la capacidad de las personas para reconocer los síntomas de las ETS, con lo que aumentarán las probabilidades de que soliciten atención o alienten a sus parejas sexuales a hacerlo.

\section{Referencias}

1. Cohen SE, Klausner JD, Engelman J, Philip S. Syphilis in the modern era: An update for physicians. Infect Dis Clin North Am. 2013; 27(4): 705-22.

2. De Andrade RS, De Freitas EM, Rocha BA, Gusmão EDS, Filho MRM, Júnior HM. Oral findings in secondary syphilis. Med Oral Patol Oral y Cir Bucal. 2018; 23(2): e138-43.

3. Ficarra G, Carlos R. Syphilis: The renaissance of an old disease with oral implications. Head Neck Pathol. 2009; 3(3): 195-206. 
4. Minicucci EM, Vieira RA, Oliveira DT, Marques SA. Oral manifestations of secondary syphilis in the elderly - A timely reminder for dentists. Aust Dent J. 2013; 58(3): 368-70.

5. Seibt CE, Munerato MC. Secondary syphilis in the oral cavity and the role of the dental surgeon in STD prevention, diagnosis and treatment: a case series study. Brazilian J Infect Dis. 2016; 20(4): 393-8.

6. Brischetto A, Gassiep I, Whiley D, Norton R. Retrospective Review of Treponema pallidum PCR and Serology Results: Are Both Tests Necessary? J Clin Microbiol. 2018; 56(5): e01782-17.

7. Organización Mundial de la Salud. Estrategia Mundial del Sector de la Salud contra Infecciones de Transmisión Sexual para 2016-2021. Hacia el fin de las ITS. [Internet]. Ginebra, Suiza: Organización Mundial de la Salud; 2016 [cited 2020 Jul 15]. Available from: https: //www.who.int/reproductivehealth/publications/rtis/ghss-stis/es/

8. Fregnani ER, Pérez-de-Oliveira ME, Parahyba CJ, Perez DE da C. Primary syphilis: An uncommon manifestation in the oral cavity. J Formos Med Assoc. 2017; 116(4): 326-7.

9. European Centre for Disease Prevention and Control. Syphilis. In: ECDC. Annual epidemiological report for 2017. [Internet]. Stockholm: ECDC; 2019 [cited 2020 Jul 15]. Available from: https: // www.ecdc.europa.eu/en/publications-data/syphilis-annual-epidemiological-report-2017

10. Ros-Vivancos C, González-Hernández M, Navarro-Gracia JF, Sánchez-Payá J, González-Torga A, Portilla-Sogorb J. Evolution of treatment of syphilis through history. Rev Esp Quimioter. 2018; 31(6): 485-92.

11. Lautenschlager S. Diagnosis of syphilis: Clinical and laboratory problems. JDDG - J Ger Soc Dermatology. 2006; 4(12): 1058-75.

12. Leão JC, Gueiros LA, Porter SR. Oral manifestations of syphilis. Clinics. 2006; 61(2): 161-6.

13. Klausner. The great imitator revealed: Syphilis. Top Antivir Med. 2019; 27(2): 71-4.

14. Leuci S, Martina S, Adamo D, Ruoppo E, Santarelli A, Sorrentino R, et al. Oral Syphilis: A retrospective analysis of 12 cases and a review of the literature. Oral Dis. 2013; 19(8): 738-46.

15. Murthy V, Vaithilingam Y, Livingstone D, Pillai A. Prosthetic rehabilitation of palatal perforation in a patient with "syphilis: The great imitator." BMJ Case Rep. 2014; 1-4.

16. Matias MDP, Jesus AO de, Resende RG, Caldeira PC, Aguiar MCF de. Diagnosing acquired syphilis through oral lesions: the 12 year experience of an Oral Medicine Center. Braz J Otorhinolaryngol. 2020; 86(3): 358-63.

17. Unemo M, Ballard R, Ison C, Lewis D, Ndowa F, Peeling R. Diagnóstico de laboratorio de las infecciones de transmisión sexual, incluida la infección por el virus de la inmunodeficiencia humana [Internet]. Ginebra, Suiza: Organización Mundial de la Salud; 2014 [cited 2020 Jul 15]. Available from: https: // www.who.int/reproductivehealth/publications/rtis/9789241505840/es/

18. Newman L, Rowley J, Hoorn S Vander, Wijesooriya NS, Unemo M, Low N, et al. Global Estimates of the Prevalence and Incidence of Four Curable Sexually Transmitted Infections in 2012 Based on Systematic Review and Global Reporting. PLoS One. 2015; 10(12): 1-17.

19. Ministerio de salud. Boletín Estadístico de Enfermedades de Declaración Obligatoria en Costa Rica del año 2015 [Internet]. 2015 [cited 2020 Jul 15]. Available from: https: //www.ministeriodesalud.go.cr/ index.php/vigilancia-de-la-salud/estadisticas-y-bases-de-datos/notificacion-individual/3167-boletinde-morbilidad-enfermedades-de-declaracion-obligatoria-2015-2/file

20. Coleman E, Fiahlo A, Brateanu A. Secondary syphilis. Cleve Clin J Med. 2017; 84(7): 510-1.

21. Peeling RW, Mabey D, Kamb ML, Chen X, David J, Benzaken AS, et al. Syphilis. Nat Rev Dis Prim. 2018; 3(17073): 49.

22. Sun HJ, Samet JM, Ohrr H, Jung HK, Il SK. Smoking and cancer risk in Korean men and women. Cancer Causes Control. 2004; 15(4): 341-8.

23. Wong TSC, Wiesenfeld D. Oral Cancer. Aust Dent J. 2018; 63: S91-9.

24. Bains MK, Hosseini-Ardehali M. Palatal perforations: Past and present. Two case reports and a literature review. Br Dent J. 2005; 199(5): 267-9. 
25. Huang S, Lu R, Yang JY, Zhou G. A nonspecific ulcer on upper lip presented as the first and sole sign of syphilis. J Infect Chemother. 2020; 26(12): 1309-12.

26. Zhang W, Mao Q, Lyu X, Hua H, Yan Z. Diagnosis of oral syphilis remains a challenge - A case report. Int J Infect Dis [Internet]. 2020; 99: 231-2. Available from: https: //doi.org/10.1016/j. ijid.2020.07.049

27. Çakmak SK, Tamer E, Karadağ AS, Waugh M. Syphilis: A great imitator. Clin Dermatol. 2019; 37(3): 182-91.

28. Calvo DF, Cassarino D, Fernandez-Flores A. Syphilitic Chancre of the Lip. Am J Dermatopathol. 2020; 42(10): e143-6.

29. Porterfield C, Brodell D, Dolohanty L, Scott G. Primary Syphilis Presenting As a Chronic Lip Ulcer. Cureus. 2020; 12(2): 1-7.

30. Workowski Kimberly A, Bolan Gail A C for DC and P. Sexually Transmitted Diseases Treatment Guidelines, 2015. MMWR Recomm Rep. 2015; 64(33): 1-137.

31. Scott CM, Flint SR. Oral syphilis - Re-emergence of an old disease with oral manifestations. Int J Oral Maxillofac Surg. 2005; 34(1): 58-63.

32. Report on global sexually transmitted infection surveillance, 2018 [Internet]. Geneva: World Health Organization; 2018. IGO; 2018 [cited 2020 Jul 15]. Available from: https: //www.who.int/reproductivehealth/publications/stis-surveillance-2018/en/

33. Rowley J, Hoorn S Vander, Korenromp E, Low N, Unemo M, Abu-Raddad LJ, et al. Chlamydia, gonorrhoea, trichomoniasis and syphilis: Global prevalence and incidence estimates, 2016. Bull World Health Organ. 2019; 97(8): 548-62.

\section{Consentimiento informado:}

Se cuenta con el consentimiento informado del paciente involucrado en el reporte de caso.

\section{Declaración de Conflictos de interés:}

Las autoras no presentan conflicto de interés en la publicación del artículo.

\section{Fuentes de financiamiento:}

No existen compromisos financieros con otras instituciones estatales o privadas que pueden afectar el contenido, resultados o conclusiones de este artículo.

\section{Nota contribución de los autores:}

1. Concepción y diseño del estudio

2. Adquisición de datos

3. Análisis de datos

4. Discusión de los resultados

5. Redacción del manuscrito

6. Aprobación de la versión final del manuscrito

YVBO ha contribuido en: $1,2,3,4,5$ y 6 .

SMBO ha contribuido en: $1,2,3,4,5$ y 6 .

\section{Nota de aceptación:}

Este artículo fue aprobado por la editora de la revista Mag. Dra. Vanesa Pereira-Prado. 